\title{
Development and characterization of a laser-induced acoustic desorption source
}

\author{
Zhipeng Huang, ${ }^{1,2}$ Tim Ossenbrüggen, ${ }^{1}$ Igor Rubinsky, ${ }^{1,3}$ \\ Matthias Schust, ${ }^{1}$ Daniel A. Horke, ${ }^{1,3}$ and Jochen Küpper ${ }^{1,2,3, *}$ \\ ${ }^{1}$ Center for Free-Electron Laser Science, Deutsches Elektronen-Synchrotron DESY, Notkestrasse 85, 22607 Hamburg, Germany \\ ${ }^{2}$ Department of Physics, Universität Hamburg, Luruper Chaussee 149, 22761 Hamburg, Germany \\ ${ }^{3}$ The Hamburg Center for Ultrafast Imaging, Universität Hamburg, Luruper Chaussee 149, 22761 Hamburg, Germany
}

(Dated: October 19, 2017)

\begin{abstract}
A laser-induced acoustic desorption source, developed for use at central facilities, such as free-electron lasers, is presented. It features prolonged measurement times and a fixed interaction point. A novel sample deposition method using aerosol spraying provides a uniform sample coverage and hence stable signal intensity. Utilizing strong-field ionization as a universal detection scheme, the produced molecular plume is characterized in terms of number density, spatial extend, fragmentation, temporal distribution, translational velocity, and translational temperature. The effect of desorption laser intensity on these plume properties is evaluated. While translational velocity is invariant for different desorption laser intensities, pointing to a non-thermal desorption mechanism, the translational temperature increases significantly and higher fragmentation is observed with increased desorption laser fluence.
\end{abstract}

\section{INTRODUCTION}

Recent years have seen the development of several techniques to control isolated neutral molecules in the gas-phase. Molecular beams of polar molecules can be dispersed with strong inhomogeneous electric fields, producing pure samples of individual conformers, cluster stoichiometries or even single quantum-states ${ }^{1-7}$ We can, furthermore, control the alignment and orientation of complex gas-phase molecules in space, $\frac{811}{11}$ allowing one to extract molecular-frame information, such as nuclear or electronic structures, from these samples $12 \mid 13$ In combination with the technological developments in free-electron laser (FEL) ultrafast x-ray sources, now providing millijoulelevel pulses of hard x-rays with sub-100 fs pulse durations, these control techniques open up the potential to image isolated biomolecules and particles with femtosecond temporal and picometer spatial resolution. 14 [17

The realization of these experiments crucially depends on a high-density source of intact molecules in the gasphase, ready for further manipulation and experiments. While for many small stable compounds this is easily achieved using thermal vaporization and seeding into a molecular beam, this approach is not feasible for thermally labile or non-volatile species - such as most larger biochemically relevant molecules, and biological species in general. Therefore, these samples, which are one of the primary driving forces behind FEL facilities, require the development of gentle vaporization techniques, that still produce a pure and high-density sample of molecules in the gas-phase. Furthermore, technical requirements for central-facility experiments, such as a well-defined and fixed interaction point and capabilities for long uninterrupted measurements times, need to be fulfilled.

One approach to achieve relatively dense ensembles of labile neutral molecules is laser-induced acoustic desorption (LIAD), which has been introduced over 30 years ago $\frac{18}{18}$ but received relatively little attention since. What sets LIAD apart from other laser-based vaporization tech- niques, such as laser desorption,,$[19$ is that it avoids any direct interaction between the desorption laser and the molecular sample, making this technique applicable to light-sensitive and labile compounds. The basic principle of LIAD is that samples get deposited on one side of an opaque substrate - often a thin metal foil - while the other side of this substrate gets irradiated with a laser pulse. This laser pulse induces an acoustic or thermal wave in the substrate, which travels through the material and leads to desorption of molecules on the front side. The physical mechanism behind this desorption process is currently very poorly understood, i. e., even the nature of the desoprtion process (thermal, acoustic, stress-induced) is not clearly established and, furthermore, it is highly dependent on the employed substrate and sample preparation method 20

Nonetheless, the LIAD technique has been used in a number of mass spectrometry studies ${ }^{21}$ Notably, the Kenttämaa group coupled LIAD to a Fourier transform ion cyclotron mass spectrometer ${ }^{24}-26$ and a quadrupole linear time-of-flight mass spectrometer. this source to study peptides and large organic compounds up to $\sim 500 \mathrm{u}$ mass. Recently, the LIAD methodology has also been applied to study the dynamics of intact aminoacids on the femtosecond and attosecond timescale using ion-yield and photoelectron spectroscopy. ${ }^{29}[31$ In a seminal paper in 2006, Peng et al. showed the applicability of LIAD to significantly larger systems and particles, successfully desorbing viruses, bacteria and cells and storing them in a quadrupole ion trap for precise mass measurements. ${ }^{22 \mid 32}$ The Campbell group furthermore established a closely related technique, termed "laser-induced forward transfer" for the gentle vaporisation of large nanoparticles.

Here, we present our new LIAD-source setup, designed for use in central facilities such as free-electron lasers or advanced-table-top-laser user facilities. It allows for prolonged measurement times through automatic sample replenishment, whilst keeping the interaction point fixed. 


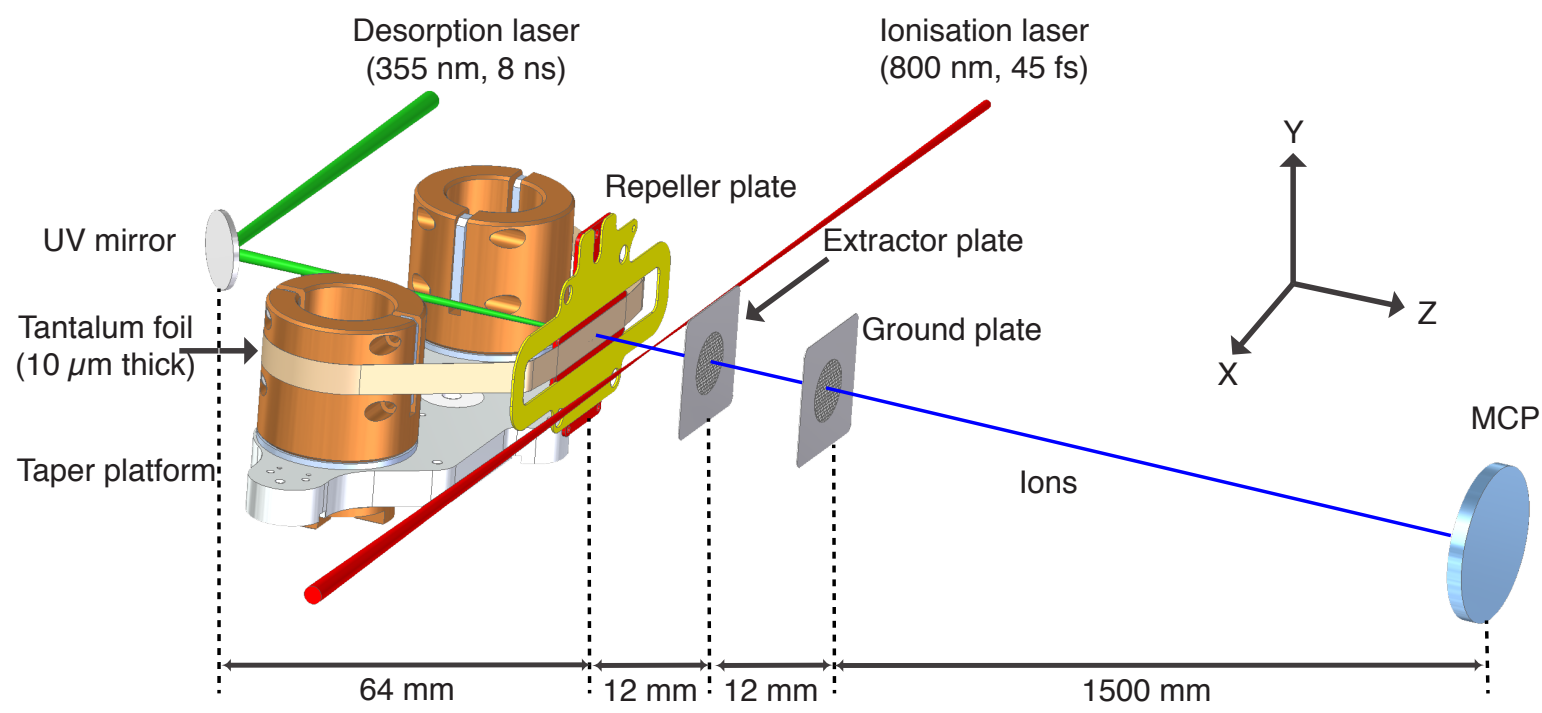

FIG. 1. LIAD setup with sample delivery based on a rotating tape drive. A taper platform contains two brass rollers to store a metal tape with applied sample on the front surface. This is fed across a metal plate (shown in red) with a small central hole for the desorption laser to pass through. This plate furthermore acts as the repeller in a time-of-flight mass spectrometer. Desorbed molecules exit the foil and are ionized by a femtosecond laser beam. Created ions are detected by a micro-channel plate (MCP) ion detector. See text for further details.

This is realized through the use of a long metal tape as the LIAD substrate, which is constantly forwarded akin to an old-fashioned cassette tape - to provide fresh sample. A reproducible layer of molecules is prepared on this foil by spraying aerosolized samples onto the band. This technique yields a stable and reproducible signal for many hours of measurement time. As a test system we use the amino acid phenylalanine and characterize the produced molecular plume using strong-field ionization, evaluating the number density, spatial extend and temporal distribution. By convoluting the initial plume temporal distribution with a Maxwell-Boltzmann velocity distribution, the forward velocity and the translational temperature in the moving frame were derived. While the velocity does not increase with desorption laser intensity, the translational temperature does increase and, furthermore, we observe enhanced fragmentation. These observations are consistent with a desorption model based on surface stress between the foil band and islands of deposited molecules, which was previously proposed 20

\section{EXPERIMENTAL METHOD}

\section{A. Experimental setup}

A schematic of our new LIAD setup is shown in Figure 1 It consists of a sample cartridge "taper platform" containing the sample of interest deposited on a long foil band and a time-of-flight mass spectrometer (TOF-MS) that can be directly coupled to the taper platform. The entire setup is housed inside a vacuum chamber, which is evacuated with a turbomolecular pump (Pfeiffer Vacuum HiPace
$700)$ to typical operating pressures of $10^{-8}$ mbar. The TOF-MS has a conventional three-plate Wiley-McLaren design, a $\sim 1.5 \mathrm{~m}$ long flight tube, and a $18 \mathrm{~mm}$ diameter microchannel plate detector for ion detection, achieving a mass resolution $m / \Delta m>1000$. The detector can be isolated from the main vacuum chamber by a gate valve to allow sample exchange without venting the detector. The detector chamber is pumped by a turbomolecular pump (Pfeiffer Vacuum TMU261) and a pressure of $10^{-9}$ mbar is typically maintained. The vacuum chamber allows optical access for the desorption and ionization lasers through anti-reflection coated windows.

The taper platform, shown in detail in Figure 1, consists of a solid aluminum baseplate, onto which two brass rollers (outer diameter $50 \mathrm{~mm}$ ) are mounted with grease-free vacuum compatible roller bearings (HWG Horst Weidner $\mathrm{GmbH}$ ). Between the two rollers the tantalum foil band containing the molecular sample is held under tension. One of the rollers is connected via an axle and rotation feedthrough to a stepper motor (Schneider LMDCE421G1A9) housed outside the vacuum to allow motorized rotation at variable speeds. The other roller is freely rotating with an adjustable resistance. At the front of the platform the foil band is fed over a stainless steel plate with rounded edges (shown in red in Figure 1) to avoid damaging the foil band and a central hole of $4 \mathrm{~mm}$ diameter to allow the desorption laser to reach the backside of the foil. This stainless steel plate can interlock with a corresponding part (shown in yellow in Figure 1 on the TOF electrode setup to ensure repeatable and correct alignment of the molecule source to the TOF electrodes. The entire taper platform is mounted on a quick release platform inside the vacuum chamber and can 
be quickly exchanged; two stainless steel dowels ensure repeatable alignment inside the vacuum chamber. During data collection the foil band is moved across the desorption laser spot by rotating the brass rollers, thereby constantly providing fresh sample. The typical speed of the foil band was on the order of $50 \mu \mathrm{m} / \mathrm{s}$, and is discussed in detail in Section III A.

Molecules are desorbed from the foil band by the third harmonic of a nanosecond Nd:YAG laser (Innolas Spitlight 600), providing $355 \mathrm{~nm}$ pulses with $\sim 8 \mathrm{~ns}$ duration at a repetition rate of $20 \mathrm{~Hz}$. These are focused onto the foil band with a $f=500 \mathrm{~mm}$ lens to a spot size of $300 \mu \mathrm{m}$ (FWHM). Once desorbed, molecules were ionized between the repeller and extractor plate of the TOF-MS through strong-field ionization, induced by 40 fs pulses from an amplified Ti:Sapphire laser (Spectra Physics Spitfire ACE). The ionization laser was focused with a $f=750 \mathrm{~mm}$ lens to a spot size of $100 \mu \mathrm{m}$ (FWHM) in the interaction region, with typical field strengths on the order of $4 \times 10^{13} \mathrm{~W} / \mathrm{cm}^{2}$. The ion current produced on the detector is read out with a digitizer (SP Devices ADQ412) and was calibrated to yield the approximate number of incident ions by first recording the current observed from a single ion hit, and using this calibration factor to convert measured currents into an approximate number of ions.

\section{B. Sample preparation}

Samples were prepared on a tantalum foil band $(\mathrm{H}$ Cross, $99.9 \%$ purity) of $10 \mu \mathrm{m}$ thickness, $10 \mathrm{~mm}$ width and approximately $1 \mathrm{~m}$ length. In order to create a stable and reproducible signal in the experiment, the deposition of a uniform thin layer of sample molecules across the entire length of the foil band is vital. Due to the length and fragility of the band, physical application of solid samples (such as rubbing or brushing on) did not yield uniform thin layers across the entire band. In order to control the sample application process more rigidly, we aerosolized sample molecules using a gas-dynamic virtual nozzle (GDVN) ${ }^{35 \mid 36}$ and deposited this aerosol onto the foil, where it rapidly dries out and sticks. The sample thickness can be controlled by adjusting the flow rate of sample through the GDVN, and thereby the aerosol production rate, or by adjusting the speed of the foil band as it moves through the aerosol beam.

In particular for the experiments presented here, we aerosolized a $0.1 \mathrm{M}$ aqueous solution of phenylalanine (Sigma-Aldrich, > 99.8\% purity, used without further purification) inside a ceramic injection-molded GDVN with a liquid inner capillary of $75 \mu \mathrm{m}$ diameter. ${ }^{36}$ A liquid flow rate of $20 \mathrm{\mu l} / \mathrm{min}$ was maintained and focused at the tip of the nozzle into a thin liquid jet by helium focusing gas at 30 bar pressure. After $\sim 1 \mathrm{~mm}$ propagation the liquid jet becomes unstable and breaks up into small droplets, producing a fine mist of aerosolized particles, which was deposited onto the foil band placed $40 \mathrm{~mm}$ away. The aerosolization and deposition process was

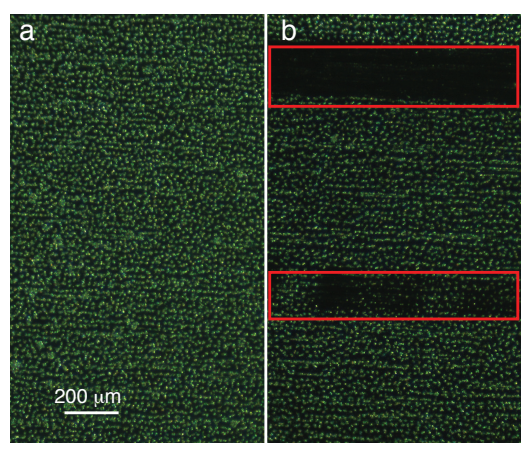

FIG. 2. Optical microscope image of phenylalanine deposited on a $10 \mu \mathrm{m}$ Ta foil using aersolization spraying. Images were taken before (a) and after (b) desorption in the LIAD setup. The latter clearly shows two regions where the desorption laser has depleted the deposited sample, as indicated by the red framed regions.

constantly monitored through a long working distance microscope. The foil band was advanced at $120 \mathrm{\mu m} / \mathrm{s}$; thus, it took approximately $140 \mathrm{~min}$ to cover the entire $1 \mathrm{~m}$ long foil band with sample. By weighing foil bands before and after application of sample, we determine an approximate surface coverage of $470 \mathrm{nmol} / \mathrm{cm}^{2}$. This should be considered an upper limit since this estimation is based purely on the weight of applied material and it is unclear to what extend water remains bound to phenylalanine and hence gets deposited on the foil band, adding to the recorded weight.

A microscope image of a foil band after sample deposition is shown in Figure 2 a, taken with a standard optical microscope (Carl Zeiss Stemi 2000). This clearly shows a uniform distribution (standard deviation of the image intensity is $\sim 2.4 \%$ ) of deposited small islands of sample across the entire surface. For comparison, we also show an image of a foil band following desorption of sample in our LIAD setup in Figure 2 b. In these images the sample was moved horizontally while the desorption laser was on, leading to a dark stripe across the image where the foil is void of sample. The upper (and darker) gap corresponds to desorption of samples with $2 \mathrm{~J} / \mathrm{cm}^{2}$ laser intensity, which removed nearly all sample from the foil. The lower one was taken with $0.7 \mathrm{~J} / \mathrm{cm}^{2}$, and some sample molecules are observed to remain on the foil band. The vertical width of these gaps is approximately $200 \mu \mathrm{m}$.

\section{RESULTS AND DISCUSSION}

\section{A. Characterizing LIAD by strong-field ionization}

In order to characterize the molecular plume desorbed from the metal foil band we utilize strong-field ionization from a focused femtosecond Ti:Sapphire laser as an universal probing scheme.29137 The observed time-of-flight mass spectrum of phenylalanine (PA) is shown in Figure 3 and compared to a literature spectrum obtained using 


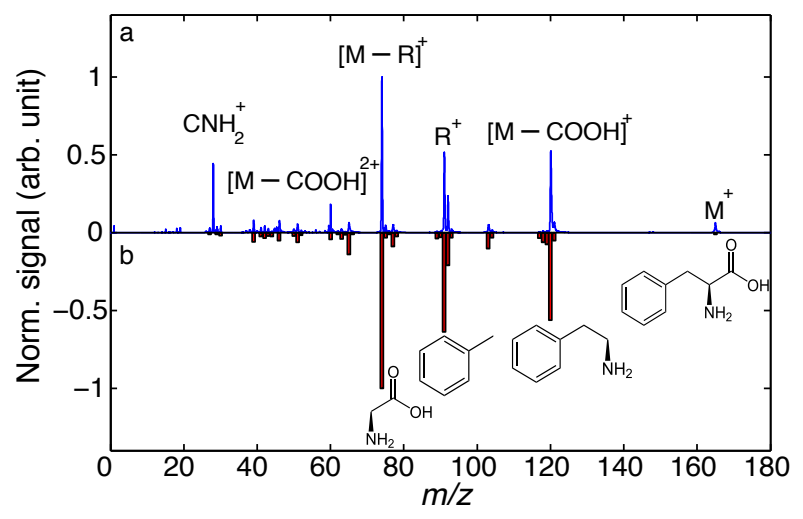

FIG. 3. Mass spectrum of phenylalanine; (a) recorded using LIAD and strong-field ionization from a femtosecond laser beam and (b) reference spectrum for electron impact ionization. ${ }^{38}$ The intensity in both spectra is normalized to the dominant mass peak at $74 \mathrm{u}$.

electron-impact ionization (EI) $\stackrel{38}{[3}$ Both spectra are normalized to the intensity of the most abundant fragment at mass $74 \mathrm{u}$, corresponding to loss of a benzyl-radical fragment from PA. It is evident that both ionization schemes strongly induce fragmentation, however we do note that using SFI a significant contribution from intact PA is observed at $165 \mathrm{u}$; this could even be enhanced using improved laser pulses ${ }^{[29}$ We observe no evidence in the mass spectrum for the production of larger clusters of PA, and hence attribute this channel to intact PA monomers desorbed from the foil. Furthermore, we observe an additional fragmentation channel in the SFI data, loss of a $\mathrm{C}-\mathrm{NH}_{2}$ group $(28 \mathrm{u})$, which is absent in the EI mass spectrum. These spectra clearly demonstrate the production of intact PA following desorption from the foil band. We do not observe the emission of any tantalum atoms or clusters from the foil band, which would easily be ionized by the SFI probe, since the ionization potential of tantalum is lower than of PA. This indicates that the desorption laser does not penetrate through the foil band nor ablate metal from the foil by other means. We further discuss the observed fragmentation in Section IIIC below.

To assess the depletion of deposited sample from the foil and to determine the required moving speed of the foil band for sample replenishment, we measured the parent ion yield as a function of the number of desorption laser shots onto the same spot. The resulting intensities are shown in Figure $4 \mathrm{a}$, where the solid line represent a power-law fit of the form $y=A \times x^{n}$, with a fitted exponent of $\mathrm{n}=-0.68 \pm 0.03$. We observe a rapid decay of signal, with signal levels falling to around $10 \%$ after 330 desorption laser shots. Similar power-law behavior has previously been observed and rationalized with the existence of several isolated desorption centers on the foil surface ${ }^{[20}$ This is consistent with our observation of many large crystalline islands on the foil surface, see Figure 2. many of which fall within the desorption laser spot size.
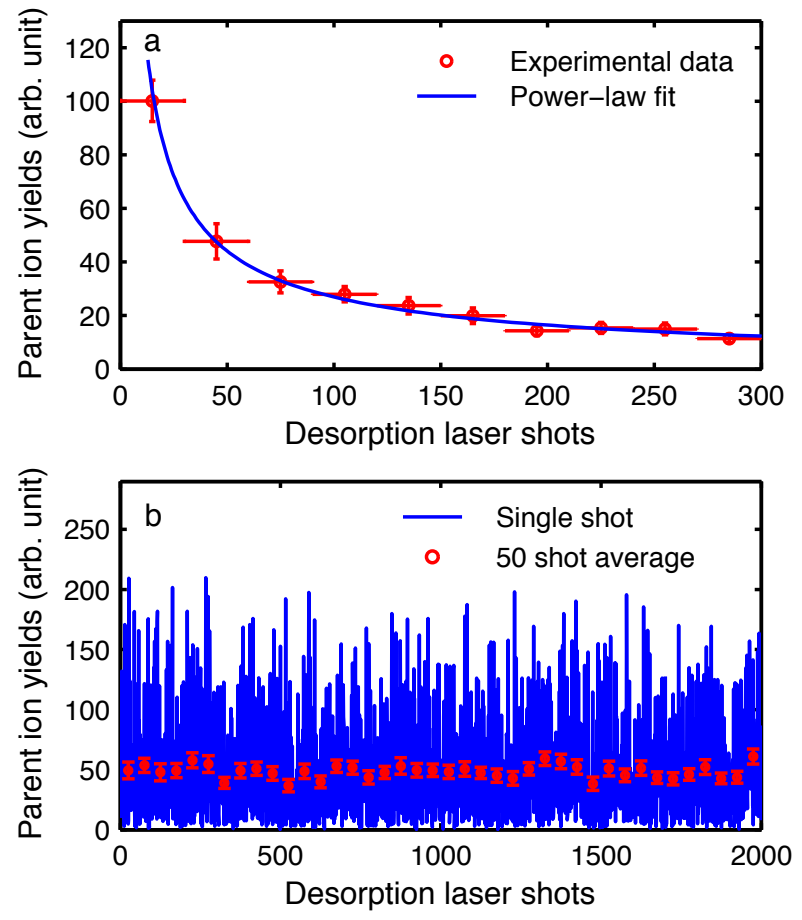

FIG. 4. (a) Parent ion yield as a function of desorption laser shot without sample replenishment. Data have been averaged over 30 shot-wide intervals, represented by the horizontal bars; the solid line corresponds to a power-law fit. (b) Parent ion signal as a function of desorption laser shot while moving the foil band at $50 \mu \mathrm{m} / \mathrm{s}$. The blue line corresponds to single-shot measurements, red markers correspond to averaged data for 50 shots, showing a standard deviation below $10 \%$.

During further data collection the foil band is continuously moved at $50 \mu \mathrm{m} / \mathrm{s}$, corresponding to a movement to a new sample spot every $\sim 120$ desorption laser shots. The corresponding shot-to-shot signal stability for the moving foil band is shown in Figure $4 \mathrm{~b}$. The signal exhibits large fluctuations with a single shot standard deviation of $70 \%$ of the mean value. No long-term drift of the overall signal levels is observed. Averaging over 50 desorption laser shots reduces the standard deviation to below 10\%, as indicated by the red markers and error bars in Figure 4. Further data points in this manuscript are typically averaged over 1200 desorption laser shots, resulting in a standard deviation of $\sim 2.5 \%$.

\section{B. Molecular plume properties}

In the following we investigate the spatial extent, density, velocity, and translational temperature of the "plume" of molecules desorbed from the foil band. We estimate absolute number densities from ion counting measurements and the known interaction volume as defined by our ionization laser, as described in Section II. In Figure 5a we show the measured number density of parent ions in the center of the desorbed plume as a function 

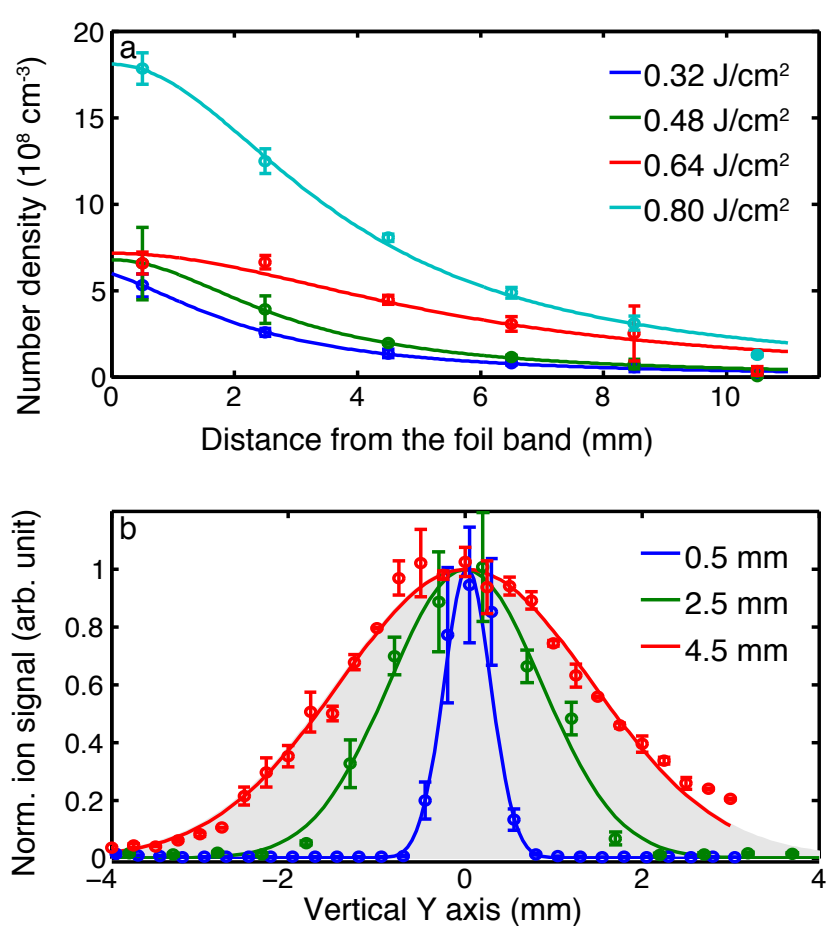

FIG. 5. (a) Parent ion number density as a function of distance from the foil band, showing inverse-square law behavior. (b) Transverse profile of the molecular plume at three distances from the foil band. Gray shading corresponds to the measured acceptance of the TOF spectrometer, such that the measurement at $4.5 \mathrm{~mm}$ does not represent the actual spatial extend of the plume, but the limits of the experimental acceptance. Solid lines correspond to Gaussian fits to the data.

of distance from the foil band. We note that the shown densities are lower limits, since their calculation assumes an ionization efficiency of 1 for SFI and considers the measured intact parent ions only, such that any fragmentation induced by the SFI probe will reduce the derived density. The obtained densities exhibit approximately an inverse-square-law behavior with distance from the desorption spot on the foil, since the expansion along the laser propagation direction is not reflected in the measurements due to the large Rayleigh length of the ionization laser $\left(z_{R} \approx 38 \mathrm{~mm}\right)$. We note that the data point closest to the foil band for the measurement at $0.64 \mathrm{~J} / \mathrm{cm}^{2}$ shows a significantly lower than expect density, which we can only explain with a lower density of molecules attached on the desorption foil band for this measurement, due to some instability during the aerosolization process.

We assess the spatial extent of the plume, i. e., the transverse profile, by translating the ionization laser in height along the $y$-axis as defined in Figure 1, across the plume of molecules. This is shown in Figure $5 \mathrm{~b}$ for three distances between the foil band surface and the interaction point. The initial profile close to the foil band is very narrow, with a FWHM of $\sim 0.6 \mathrm{~mm}$ after $0.5 \mathrm{~mm}$ of free flight. The plume of desorbed molecules then rapidly spreads out, reaching a FWHM of around $2 \mathrm{~mm}$

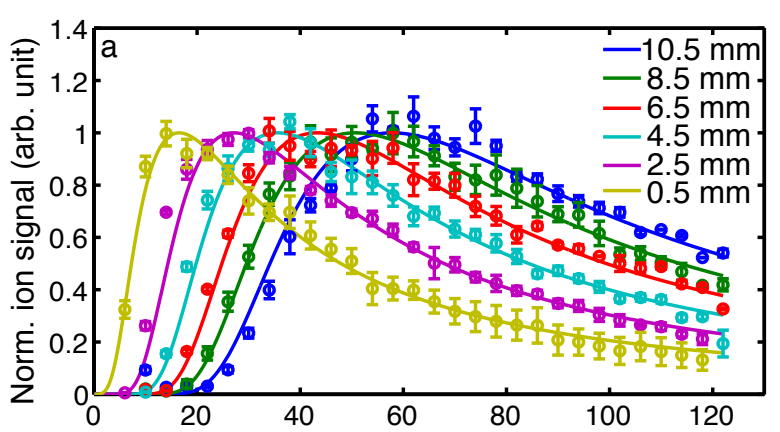

Delay between ionization and desorption laser $(\mu \mathrm{s})$

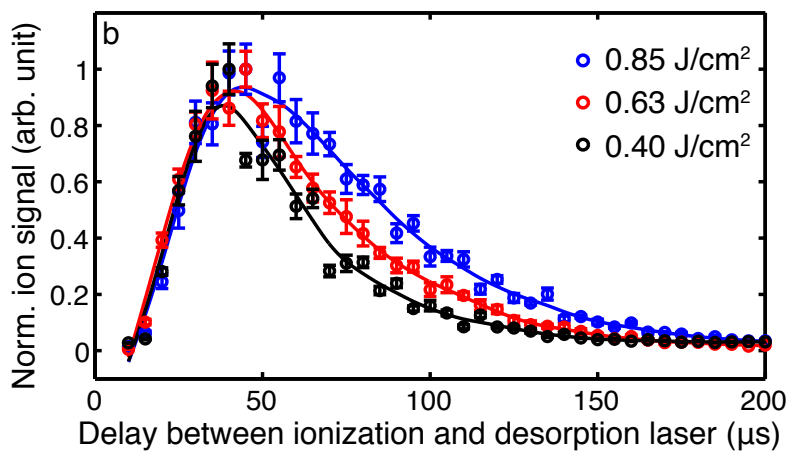

FIG. 6. (a) Normalized temporal profiles of intact parent ions following desorption with $0.8 \mathrm{~mJ} / \mathrm{cm}^{2}$, recorded for different distances from the foil band. Solid lines correspond to a fit with a Maxwell-Boltzmann distribution convoluted with the desorption time distribution. (b) Normalized temporal profiles of intact parent ions for different desorption laser intensities and otherwise identical settings, obtained at $z=6.5 \mathrm{~mm}$. While the most probable velocity is approximately constant, the larger desorption laser fluence clearly leads to a much broader velocity distribution.

after $2.5 \mathrm{~mm}$ propagation and within $4.5 \mathrm{~mm}$ of free flight the extent of the plume exceeds the spatial acceptance of the TOF ion optics (indicated by the gray shading in Figure 5b), such that no accurate data can be measured at larger separations. This rapid diffusion of the plume in space is consistent with the fast drop in density observed as the distance between the foil band and the interaction point is increased, Figure 5a, and indicates rapid diffusion of the molecular plume in space following desorption from a well-defined spot defined by the desorption laser profile.

To investigate the longitudinal extend and velocity of the plume of desorbed molecules we measure mass spectra as a function of delay between the desorption and ionization lasers, and at different distances from the foil band. Results for the intact-parent-ion yield following desorption with a fluence of $0.8 \mathrm{~mJ} / \mathrm{cm}^{2}$ are shown in Figure 6a. Similar data for other desorption fluences are shown in the supplementary data. It is very clear that even when the interaction point is very close to the foil band a broad temporal profile is observed, lasting several tens of $\mu \mathrm{s}$, much broader than the $8 \mathrm{~ns}$ duration of the desorption-laser pulse. At larger distances from the foil band these distributions widen considerable more, 
demonstrating that during free flight through the vacuum chamber the plume spreads out also in the longitudinal direction. We identify two physical origins for the observed profiles and their temporal evolution; (i) the desorption process itself that does not release molecules at one instant in time, but with a certain temporal and kinetic energy distribution and (ii) the propagation of molecules in free flight with a certain finite translational velocity distribution. Whereas (i) contains information about the physical desorption mechanism from the foil, the translational velocity spread from (ii) corresponds to the translational temperature in the moving frame of the molecules.

Assuming an equilibrated thermal distribution of molecules in the moving frame, we can model the velocity distributions with a modified Maxwell-Boltzmann distribution, where we have introduced the parameter $v_{0, z}$ as a velocity offset (or stream velocity) in the $z$ direction. This accounts for the forward momentum by the desorption process, $39 \mid 40$

$$
f(\vec{v})=\left(\frac{m}{2 \pi k_{\mathrm{B}} T}\right)^{3 / 2} e^{\frac{-m\left(v_{x}^{2}+v_{y}^{2}+\left(v_{z}-v_{0, z}\right)^{2}\right)}{2 k_{\mathrm{B}} T}} \mathrm{~d} v_{x} \mathrm{~d} v_{y} \mathrm{~d} v_{z} .
$$

Here, $m$ denotes the molecular mass, $v_{x}, v_{y}$, and $v_{z}$ the velocity components along the axes as defined in Figure 1 . $k_{\mathrm{B}}$ the Boltzmann constant and $T$ the equilibrium translational temperature of the plume in the moving frame of reference. We can simplify this expression further by considering the actual volume of the plume probed in the experiment. For these measurements the ionization laser was placed vertically in the transverse center of the plume, $y=0$. Due to the small spot size of the laser $(100 \mu \mathrm{m}), v_{y}=0$ can be assumed for the probed molecules. Similar arguments hold for the other transverse direction, $x$. While the Rayleigh range of the ionization laser $\left(z_{R} \approx 38 \mathrm{~mm}\right)$ is non-negligible compared to the plume dimensions, the focal point was located in the center of the plume and hence the average velocity in the $x$ direction is also zero. Taking these simplifications into account and re-normalizing the distribution we obtain

$$
f(v)=f\left(v_{z}\right)=\sqrt{\frac{m}{2 \pi k_{\mathrm{B}} T}} e^{\frac{-m\left(v_{z}-v_{0, z}\right)^{2}}{2 k_{\mathrm{B}} T}} \mathrm{~d} v_{z} .
$$

Since the experiment does not measure velocities directly, but rather the time $t$ it takes molecules to travel a certain distance $l$, we can substitute for $v_{z}=\frac{l}{t-t_{0}}$, where $t_{0}$ accounts for any temporal offset from the desorption process.

$$
f(t)= \begin{cases}0 & \text { if } t \leq t_{0} \\ \frac{l}{\left(t-t_{0}\right)^{2}} \sqrt{\frac{m}{2 \pi k_{\mathrm{B}} T}} e^{\frac{-m\left(\frac{l}{t-t_{0}}-v_{0}, z\right.}{2 k_{\mathrm{B}} T}} \mathrm{~d} t & \text { if } t>t_{0}\end{cases}
$$

While this simplified Maxwell-Boltzmann picture describes the velocity distribution resulting from the finite translational temperature of the molecular plume, it does not take into account the broad distribution of desorption
TABLE I. Measured translational velocities and temperatures in the moving frame for different desorption laser intensities.

\begin{tabular}{ccc}
\hline \hline Desorp. Fluence $\left(\mathrm{J} / \mathrm{cm}^{2}\right)$ & $T(\mathrm{~K})$ & $v_{0, z}(\mathrm{~m} / \mathrm{s})$ \\
\hline 0.32 & 594 & 233 \\
0.48 & 679 & 234 \\
0.64 & 715 & 265 \\
0.80 & 758 & 224 \\
\hline \hline
\end{tabular}

times from the foil band. Since so far no quantitative model is available to describe this desorption process accurately, we take the experimental data measured closest to the foil band, here $0.5 \mathrm{~mm}$, as a measure of the desorption time distribution. While this distribution has already been influenced by a short free-flight propagation, it is nonetheless the one that most closely resembles the actual time-dependence of the desorption process. Given the measured plume velocities, it takes around $2 \mu$ s for the propagation of $0.5 \mathrm{~mm}$, whereas the observed temporal distributions are significantly wider, justifying this assumption.

To fit the measured data from Figure 6 a, we numerically convolute this experimental temporal desorption distribution with the Maxwell-Boltzmann model of the free-flight propagation. We then perform a global fit of the data for all propagation distances $l$ simultaneously using a common temperature $T$ and offset velocity $v_{0, z}$, while we introduce only a single linear scaling parameter for the different data sets. The latter essentially accounts for the drop in intensity along the probed center-line of the plume. The results of this fit for a desorption laser fluence of $0.8 \mathrm{~mJ} / \mathrm{cm}^{2}$ are shown as solid lines in Figure $6 \mathrm{a}$, with data for other laser fluences provided in the supplementary information. The obtained translational temperatures and forward velocities are summarized in Table I

We observe a strong, nearly linear, dependence of the translational temperature of the molecular plume on the fluence of the desorption laser. Even at the lowest fluence used a translational temperature of nearly $600 \mathrm{~K}$ is obtained. In the current experimental setup using strong-field ionization we cannot measure the internal (vibrational or rotational) temperature directly. However, given the large density of states in systems such as phenylalanine, and the microsecond timescales of the desorption process, we can assume a large degree of thermalization between the different degrees of freedom. Thus the measured translational temperatures can be considered as a good indicator of the internal temperature of desorbed molecules.

Unlike the temperature, the observed forward velocity appears to be approximately constant for the different desorption laser fluences. The slightly elevated velocity for the measurement at $0.64 \mathrm{~J} / \mathrm{cm}^{2}$ could be due to instabilities in the sample preparation for this measurement, as mentioned above. Similar observations of identical forward velocity have been previously reported ${ }^{20|25|}$ This 

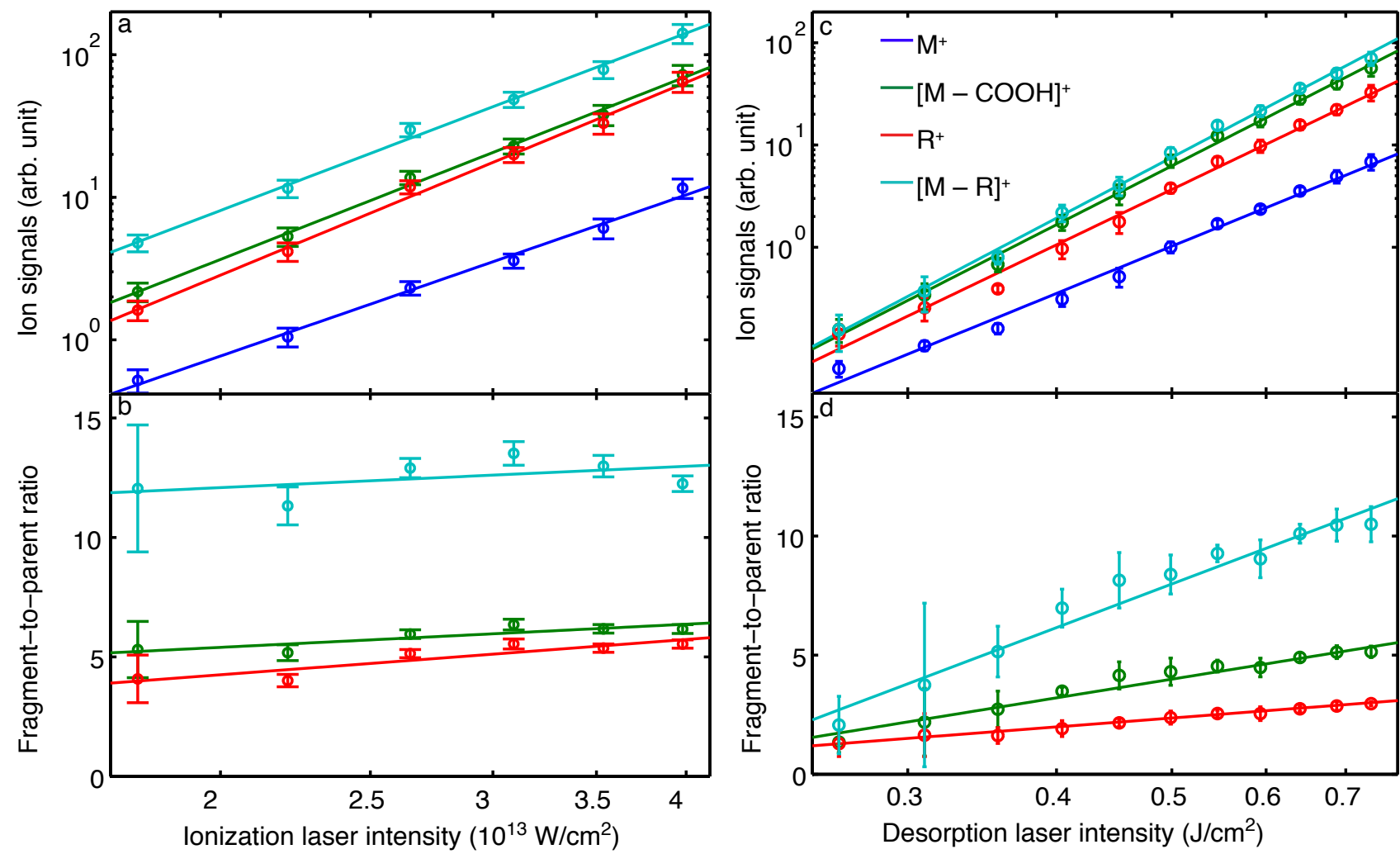

FIG. 7. Ion-yield signals (a, c) and fragment-to-parent ratios (b, d) as a function of ionization laser intensity (a, b) and desorption laser intensity $(c, d)$. Color coding for all graphs is given in panel c; see Figure 3 for assignment of the mass peaks. Solid lines correspond to power-law fits.

invariability of the velocity with desorption laser fluence suggests that this might be determined by material properties of the substrate and the molecular sample.

Figure 6b shows the yield of intact parent ions as a function of desorption laser-ionization laser delay for different desorption fluences. While the peaks of the distribution overlap in time the distribution is significantly broader for higher fluences. These observations fully support our finding of a constant translational velocity, but increasing translational temperature as the desorption laser fluence is increased (vide supra).

\section{Molecular fragmentation}

In how far the observed fragmentation is due to the desorption or the strong-field ionization process is hard to assess from the mass spectra in Figure 3 alone. In order to disentangle these contributions, we collect mass spectra for different ionization and desorption laser intensities.

Figure 7 a shows the ion yield for the PA parent and the three dominant fragments as a function of ionization laser intensity, with all ion channels showing a steep increase with increasing laser intensity. These data were fit with a power-law dependence of the form $A \times x^{n}$. Figure $7 \mathrm{~b}$ further shows the ratio of fragment-to-parent ion intensities for the three dominant fragments, i. e., comparing the relative intensities of the two respective channels. We observe only a very slight increase in fragmentation as the laser intensity increases, in good agreement with previous studies suggesting that strong-field ionization induced fragmentation is very sensitive to the employed pulse duration, but not the intensity!

Figure 7 c shows the dependence of ion yields on the intensity of the desorption laser and Figure $7 \mathrm{~d}$ the corresponding fragment-to-parent ratios. The overall measured ion intensities are again well described by a power-law fit and show a steep increase for higher intensities, especially noticeable for fragment ions. This is confirmed by the fragment-to-parent ratios, which also significantly increase with laser intensity, indicating enhanced fragmentation. Thus, the desorption-laser interaction clearly induces fragmentation, either directly during the desorption process or thereafter, but prior to ionization, i. e., as molecules travel through the vacuum chamber toward the interaction point. To test the latter, we recorded mass spectra at different distances behind the foil band, changing the laser-laser delay such that we always probe the highest density part of the molecular plume, i. e., we follow the center of the plume as it travels through the vacuum chamber. This data is shown in Figure 8 a, collected for distances of $0.5-10.5 \mathrm{~mm}$ between the foil band and the 

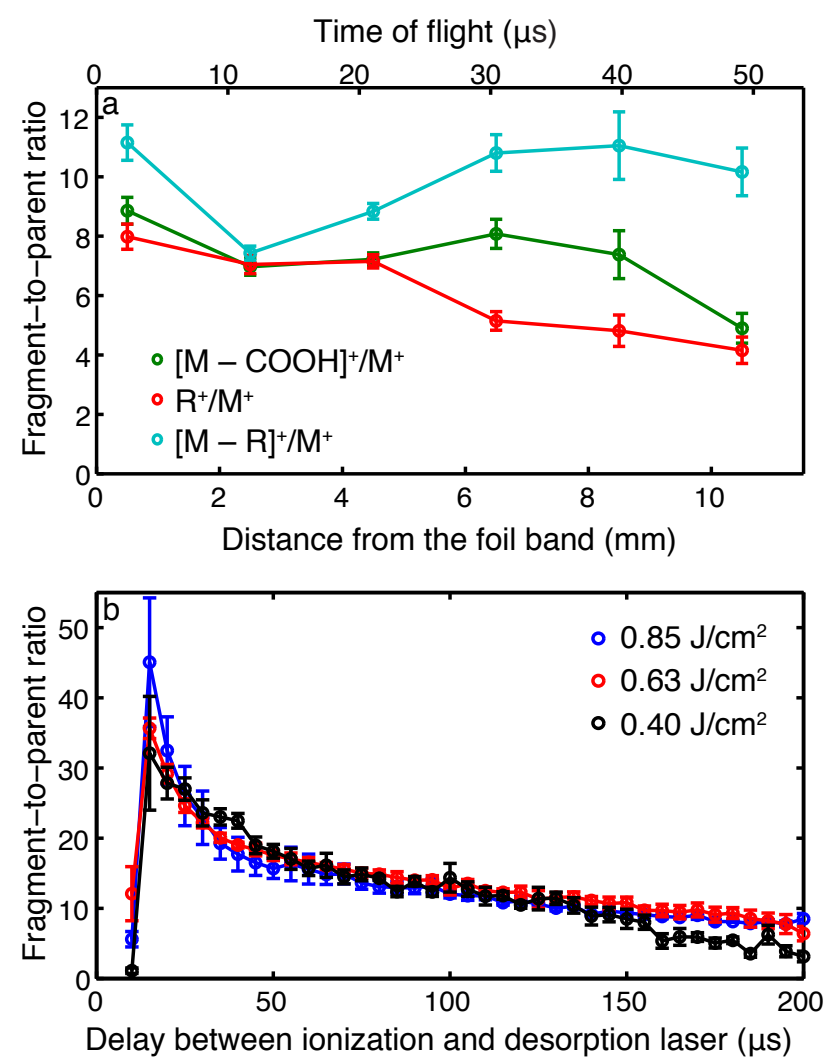

FIG. 8. (a) Fragment-to-parent ratio recorded at the peak of the molecular plume density for different distances behind the foil band. No significant increase in fragmentation is observed as the plume travels through the vacuum chamber. (b) Fragment-to-parent ratio throughout the molecular plume recorded $6.5 \mathrm{~mm}$ behind the foil for different desorption laser intensities. Molecules desorbed shortly after the arrival of the desorption laser show significantly higher fragmentation than molecules desorbed later.

interaction point, which corresponds to flight times of around 0-50 $\mathrm{\mu s}$. Over this distance we observe no significant increase in fragmentation. This indicates that the observed fragmentation occurs on much faster timescales, i. e., most likely during the desorption process itself, either while molecules are still attached to the metal substrate or very shortly after desorption into the gas-phase.

We now consider the distribution of fragments within a single plume coming from the foil band, i.e., if the fragmentation changes depending on which part of the plume is observed. This is shown in Figure $8 \mathrm{~b}$, where we plot the fragment-to-parent ratio for the most abundant molecular fragment as a function of desorption-laser-toionization-laser delay for a fixed distance from the foil band, i. e., $6.5 \mathrm{~mm}$. We observe an initial peak in the fragment-to-parent ratio at the onset of desorption, i. e., the "front" part of the molecular plume, which then decreases on a timescale of tens of microseconds. These timescales are consistent with thermal processes, in particular we associate the observed distribution with the rapid heating of the foil band by the nanosecond laser pulse, causing increased fragmentation, followed by slow dissipation of the thermal energy, i. e., cooling down of the front surface and, hence, reduced fragmentation.

Further evidence that the fragmentation occurs during the desorption process and that it is of a thermal nature comes from the comparison of the fragment-to-parent ratios throughout the plume for different desorption laser fluences, also shown in Figure 8 b. These clearly show that the highest degree of fragmentation occurs for the most intense desorption laser pulse. This is also consistent with the higher translational temperatures derived for these conditions. Once the foil band cools down, i. e., at longer desorption-laser-to-ionization-laser delays, the fragment-to-parent ratio approaches an asymptotic value independent of initial desorption conditions.

\section{Nature of the desorption process}

Several possible mechanisms have been suggested in the literature for the underlying physical processes occurring in the LIAD process.2013441142 It is important to note that the experimental conditions for the different published LIAD-based molecule sources in the literature are very different; pulsed ${ }^{20|29|}$ and continuous ${ }^{31 \mid 43}$ desorption lasers are used and sample preparation methods vary greatly, from the thick sample layer used here of $\sim 500 \mathrm{nmol} / \mathrm{cm}^{2}, 43 \mid 44$ to intermediate thicknesses of tens of $\mathrm{nmol} / \mathrm{cm}^{2}, 25 \mid 26$ to near-monolayer coverage in other studies.20 As such, we do not aim to provide a general model for the LIAD mechanisms, but seek to explain our observations and compare these with previous studies where applicable.

One of the suggested desorption mechanisms, and indeed the origin of the term "acoustic desorption", 4142 is the direct momentum transfer from a shock wave induced by the desorption laser in the foil band to the sample molecules. The data presented here firmly rules out this mechanism for our molecule source. We observe a slow rise in molecular signal on the order of $\sim 10 \mu \mathrm{s}$, see Figure 6 , which is not compatible with molecules being "shaken off", by an impulse traveling through the foil, as this should lead to a sharp sudden onset of signal as the impulse reaches the front surface, followed by an immediate drop as the impulse is reflected on the surface. Additionally one might expect to observe a periodic revival of signal as the impulses bounces back and forth within the metal foil. We observe no evidence for this behavior in our data. Furthermore, the travel time for a mechanical wave through a $10 \mu \mathrm{m}$ tantalum foil is approximately 2 ns, 45 significantly shorter than the delay we observe between the desorption laser impacting on the foil and molecules being desorbed. A purely acoustic desorption mechanism would, furthermore, not explain the observed increase in fragmentation for increased desorption laser fluences. Similar observations have been made previously for a pulsed LIAD setup, and the "shake off" mechanism 
similarly discredited 20

The other conceptually simple mechanism behind the observed desorption of molecules is a simple thermal one; the incident laser pulse heats up the material from the backside and this thermal energy then diffuses to the front of the foil where it heats up molecules and they eventually desorb. However, the observation that the velocity and, therefore, the kinetic energy of desorbed molecules is independent of the incident desorption laser power and thus surface temperature is not compatible with a purely-thermal desorption model.

The observation that the kinetic energy of desorbed molecules is independent of desorption laser fluence indicates that this is determined by material properties of the foil substrate and/or the molecular sample. This observation, along with the increase in translational temperature in the moving frame, is consistent with a desorption model proposed by Zinovev et al. ${ }^{20}$ They explain the LIAD process by an introduction of surface stress between the substrate and the molecular sample - located in isolated islands on the substrate - due to the acoustic and/or thermal wave created by the desorption laser. This surface stress can lead to elastic deformation, decomposition, and cracking of sample islands on the foil band and, eventually, to desorption of molecules. In this conceptual model the kinetic energy transferred to a desorbing molecule is independent of the total incident laser power, and rather depends on the intrinsic characteristics of a given sample island and substrate. A higher laser fluence leads to the introduction of more surface stress and the formation of more cracks and deformation sites, leading to an increase in molecular signal, but does not influence the amount of kinetic energy per molecule. At the same time we note that due to thermal conductivity the higher temperature of the substrate reached for higher desorption laser fluences will also heat up deposited sample molecules due to thermal conduction, leading to internally hotter molecules, increased fragmentation as well as higher translational temperatures.

While it is difficult to theoretically model the amount of energy transferred to each desorbed molecule, Zinovev et al. provide a simple formula to estimate the energy per analyte molecule based on material properties and thermal stress theory ${ }^{20}$ Based on this we estimate $25-100 \mathrm{meV}$ of energy per molecule for temperature differences of $\Delta T=100-200 \mathrm{~K}{ }^{[46}$ This is well within the range of the measured kinetic energy per molecule which is, based on the average velocity observed, around $50 \mathrm{meV}$. Thus, our data is fully supportive of the proposed surface stress model.

\section{CONCLUSION}

We presented an advanced LIAD source for the preparation of gas-phase samples of labile molecules, designed for the use at central-facility light sources such as free-electron lasers. It features a prolonged continuous measurement time through automatic sample replenishment using a long foil band, as well as a fixed interaction point. Uniform sample preparation on the long substrate was achieved using an aerosol spraying method based on thin liquid jets. We have characterized the new source using phenylalanine as a sample molecule and strong-field ionization as a universal probe method. We observe a significant fraction of intact molecules being desorbed from the foil, with number densities around $2 \times 10^{8} \mathrm{~cm}^{-3}$ close to the foil band. Due to fragmentation processes induced by the femtosecond laser probe, this should be treated as a lower limit. The spatial extend of the molecular plume rapidly spreads out from the point of desorption, leading to a corresponding drop in density with increasing distance from the foil band. The plume forward translational velocity and temperature in the moving frame are derived by convoluting a Maxwell-Boltzmann velocity distribution with the initial temporal profile near the foil band. The forward velocity, and hence kinetic energy, of molecules desorbed from the foil does not depend on the desorption laser intensity. In contrast to this, the translational temperature clearly increases with increasing desorption intensity. We investigated the fragmentation processes and observe increased fragmentation at higher desorption laser intensity, consistent with the translational temperature behavior. Furthermore, we show that the amount of fragmentation depends on the time of desorption from the foil: shortly after the laser pulse molecules are observed to be hottest, and subsequently they cool down on thermal timescales (10s of $\mu \mathrm{s})$ as the substrate itself cools down. These observations are fully supported by the previously proposed surface-stress model of the LIAD process.

Our characterization measurements show that our new source produces a stable high-density signal of intact molecules in the gas-phase. With automatic sample replenishment it provides very long continuous measurement times. The produced molecular plume is well suited for further gas-phase experiments and manipulation, and work is currently underway towards integrating this source into a buffer-gas-cooling setup for the production of cold molecules, $\stackrel{47}{ }$ which can then be further manipulated using electric fields! 7 One could also envision to make use of this desorption technique for the entrainment of molecules into supersonic beams, similar to matrix-assisted laser desorption approaches $\frac{37}{37}$ Furthermore, we are working towards a better understanding of the desorption mechanism and its dependence on the sample layer thickness and sample itself in order to successfully apply our new source to the gentle vaporization of more labile biological samples.

\section{ACKNOWLEDGMENTS}

In addition to DESY, this work has been supported by the European Research Council under the European Union's Seventh Framework Programme (FP7/2007-2013) through the Consolidator Grant COMOTION (ERC- 
614507-Küpper), by the excellence cluster "The Hamburg Center for Ultrafast Imaging - Structure, Dynamics and Control of Matter at the Atomic Scale" of the Deutsche Forschungsgemeinschaft (CUI, DFG-EXC1074), and by the Helmholtz Gemeinschaft through the "Impuls- und Vernetzungsfond". Z. H. gratefully acknowledges a scholarship of the Joachim-Herz-Stiftung and support from the PIER Helmholtz Graduate School.
* jochen.kuepper@cfel.de https://www.controlled-moleculeimaging.org

${ }^{1}$ Frank Filsinger, Undine Erlekam, Gert von Helden, Jochen Küpper, and Gerard Meijer, "Selector for structural isomers of neutral molecules," Phys. Rev. Lett. 100, 133003 (2008) arXiv:0802.2795 [physics]

2 Frank Filsinger, Jochen Küpper, Gerard Meijer, Jonas L. Hansen, Jochen Maurer, Jens H. Nielsen, Lotte Holmegaard, and Henrik Stapelfeldt, "Pure samples of individual conformers: the separation of stereo-isomers of complex molecules using electric fields," Angew. Chem. Int. Ed. 48, 6900-6902 (2009).

Sebastian Trippel, Yuan-Pin Chang, Stephan Stern, Terry Mullins, Lotte Holmegaard, and Jochen Küpper, "Spatial separation of state- and size-selected neutral clusters," Phys. Rev. A 86, 033202 (2012) arXiv:1208.4935 [physics]

${ }^{4}$ Jens H. Nielsen, Paw Simesen, Christer Z. Bisgaard, Henrik Stapelfeldt, Frank Filsinger, Bretislav Friedrich, Gerard Meijer, and Jochen Küpper, "Stark-selected beam of ground-state OCS molecules characterized by revivals of impulsive alignment," Phys. Chem. Chem. Phys. 13, 1897118975 (2011), arXiv:1105.2413 [physics]

Daniel A. Horke, Yuan-Pin Chang, Karol Długołęcki, and Jochen Küpper, "Separating para and ortho water," Angew. Chem. Int. Ed. 53, 11965-11968 (2014), arXiv:1407.2056 |physics]

- Sebastiaan Y T van de Meerakker, Hendrick L Bethlem, Nicolas Vanhaecke, and Gerard Meijer, "Manipulation and control of molecular beams," Chem. Rev. 112, 4828-4878 (2012).

Yuan-Pin Chang, Daniel A. Horke, Sebastian Trippel, and Jochen Küpper, "Spatially-controlled complex molecules and their applications," Int. Rev. Phys. Chem. 34, 557-590 (2015), arXiv:1505.05632 [physics]

$\checkmark$ Lotte Holmegaard, Jens H. Nielsen, Iftach Nevo, Henrik Stapelfeldt, Frank Filsinger, Jochen Küpper, and Gerard Meijer, "Laser-induced alignment and orientation of quantum-state-selected large molecules," Phys. Rev. Lett. 102, 023001 (2009), arXiv:0810.2307 [physics].

${ }^{9}$ Frank Filsinger, Jochen Küpper, Gerard Meijer, Lotte Holmegaard, Jens H. Nielsen, Iftach Nevo, Jonas L. Hansen, and Henrik Stapelfeldt, "Quantum-state selection, alignment, and orientation of large molecules using static electric and laser fields," J. Chem. Phys. 131, 064309 (2009), arXiv:0903.5413 [physics].

10 Sebastian Trippel, Terry Mullins, Nele L. M. Müller, Jens S. Kienitz, Karol Długołęcki, and Jochen Küpper, "Strongly aligned and oriented molecular samples at a $\mathrm{kHz}$ repetition rate," Mol. Phys. 111, 1738 (2013), arXiv:1301.1826 [physics]

${ }^{11}$ Henrik Stapelfeldt and Tamar Seideman, "Colloquium: Aligning molecules with strong laser pulses," Rev. Mod. Phys. 75, 543-557 (2003)

I2 Christer Z. Bisgaard, Owen J. Clarkin, Guorong Wu, Anthony M. D. Lee, Oliver Geßner, Carl C. Hayden, and
Albert Stolow, "Time-resolved molecular frame dynamics of fixed-in-space $\mathrm{CS}_{2}$ molecules," Science 323, 1464-1468 (2009).

13 Lotte Holmegaard, Jonas L. Hansen, Line Kalhøj, Sofie Louise Kragh, Henrik Stapelfeldt, Frank Filsinger, Jochen Küpper, Gerard Meijer, Darko Dimitrovski, Mahmoud Abu-samha, Christian P. J. Martiny, and Lars Bojer Madsen, "Photoelectron angular distributions from strongfield ionization of oriented molecules," Nat. Phys. 6, 428 (2010), arXiv:1003.4634 [physics]

${ }_{14}$ M Marvin Seibert, Tomas Ekeberg, Filipe R N C Maia, Martin Svenda, Jakob Andreasson, Olof Jönsson, Duško Odić, Bianca Iwan, Andrea Rocker, Daniel Westphal, Max Hantke, Daniel P Deponte, Anton Barty, Joachim Schulz, Lars Gumprecht, Nicola Coppola, Andrew Aquila, Mengning Liang, Thomas A White, Andrew Martin, Carl Caleman, Stephan Stern, Chantal Abergel, Virginie Seltzer, Jean-Michel Claverie, Christoph Bostedt, John D Bozek, Sébastien Boutet, A Alan Miahnahri, Marc Messerschmidt, Jacek Krzywinski, Garth Williams, Keith O Hodgson, Michael J Bogan, Christina Y Hampton, Raymond G Sierra, Dmitri Starodub, Inger Andersson, Saša Bajt, Miriam Barthelmess, John C H Spence, Petra Fromme, Uwe Weierstall, Richard Kirian, Mark Hunter, R Bruce Doak, Stefano Marchesini, Stefan P Hau-Riege, Matthias Frank, Robert L Shoeman, Lukas Lomb, Sascha W Epp, Robert Hartmann, Daniel Rolles, Artem Rudenko, Carlo Schmidt, Lutz Foucar, Nils Kimmel, Peter Holl, Benedikt Rudek, Benjamin Erk, André Hömke, Christian Reich, Daniel Pietschner, Georg Weidenspointner, Lothar Strüder, Günter Hauser, Hubert Gorke, Joachim Ullrich, Ilme Schlichting, Sven Herrmann, Gerhard Schaller, Florian Schopper, Heike Soltau, Kai-Uwe Kühnel, Robert Andritschke, Claus-Dieter Schröter, Faton Krasniqi, Mario Bott, Sebastian Schorb, Daniela Rupp, Marcus Adolph, Tais Gorkhover, Helmut Hirsemann, Guillaume Potdevin, Heinz Graafsma, Björn Nilsson, Henry N Chapman, and Janos Hajdu, "Single mimivirus particles intercepted and imaged with an x-ray laser," Nature 470, 78 (2011)

15 Richard Neutze, Remco Wouts, David van der Spoel, Edgar Weckert, and Janos Hajdu, "Potential for biomolecular imaging with femtosecond X-ray pulses," Nature 406, 752757 (2000).

${ }^{16}$ Rebecca Boll, Dennis Anielski, Christoph Bostedt, John D. Bozek, Lauge Christensen, Ryan Coffee, Sankar De, Piero Decleva, Sascha W. Epp, Benjamin Erk, Lutz Foucar, Faton Krasniqi, Jochen Küpper, Arnaud Rouzée, Benedikt Rudek, Artem Rudenko, Sebastian Schorb, Henrik Stapelfeldt, Mauro Stener, Stephan Stern, Simone Techert, Sebastian Trippel, Marc J. J. Vrakking, Joachim Ullrich, and Daniel Rolles, "Femtosecond photoelectron diffraction on laseraligned molecules: Towards time-resolved imaging of molecular structure," Phys. Rev. A 88, 061402(R) (2013).

17 Jochen Küpper, Stephan Stern, Lotte Holmegaard, Frank Filsinger, Arnaud Rouzée, Artem Rudenko, Per Johnsson, 
Andrew V. Martin, Marcus Adolph, Andrew Aquila, Saša Bajt, Anton Barty, Christoph Bostedt, John Bozek, Carl Caleman, Ryan Coffee, Nicola Coppola, Tjark Delmas, Sascha Epp, Benjamin Erk, Lutz Foucar, Tais Gorkhover, Lars Gumprecht, Andreas Hartmann, Robert Hartmann, Günter Hauser, Peter Holl, Andre Hömke, Nils Kimmel, Faton Krasniqi, Kai-Uwe Kühnel, Jochen Maurer, Marc Messerschmidt, Robert Moshammer, Christian Reich, Benedikt Rudek, Robin Santra, Ilme Schlichting, Carlo Schmidt, Sebastian Schorb, Joachim Schulz, Heike Soltau, John C. H. Spence, Dmitri Starodub, Lothar Strüder, Jan Thøgersen, Marc J. J. Vrakking, Georg Weidenspointner, Thomas A. White, Cornelia Wunderer, Gerard Meijer, Joachim Ullrich, Henrik Stapelfeldt, Daniel Rolles, and Henry N. Chapman, "X-ray diffraction from isolated and strongly aligned gas-phase molecules with a free-electron laser," Phys. Rev. Lett. 112, 083002 (2014), arXiv:1307.4577 [physics|

18 Buko Lindner and Ulrich Seydel, "Laser desorption mass spectrometry of nonvolatiles under shock wave conditions," Anal. Chem. 57, 895-899 (1985)

19 M S de Vries and P Hobza, "Gas-phase spectroscopy of biomolecular building blocks," Annu. Rev. Phys. Chem. 58, 585-612 (2007)

20 Alexander V Zinovev, Igor V Veryovkin, Jerry F Moore, and Michael J Pellin, "Laser-driven acoustic desorption of organic molecules from back-irradiated solid foils," Anal. Chem. 79, 8232-8241 (2007).

${ }^{21}$ V V Golovlev, S L Allman, W R Garrett, N I Taranenko, and C H Chen, "Laser-induced acoustic desorption," Int. J. Mass Spectrom. Ion Proc. 169-170, 69-78 (1997).

${ }^{22}$ Wen-Ping Peng, Yi-Chang Yang, Ming-Wei Kang, Yan-Kai Tzeng, Zongxiu Nie, Huan-Cheng Chang, Wen Chang, and Chung-Hsuan Chen, "Laser-induced acoustic desorption mass spectrometry of single bioparticles," Angew. Chem. Int. Ed. 45, 1423-1426 (2006)

${ }^{23}$ Leonard Nyadong, John P Quinn, Chang S Hsu, Christopher L Hendrickson, Ryan P Rodgers, and Alan G Marshall, "Atmospheric pressure laser-induced acoustic desorption chemical ionization mass spectrometry for analysis of saturated hydrocarbons," Anal. Chem. 84, 7131-7137 (2012)

${ }^{44}$ James Pérez, Luis E Ramírez-Arizmendi, Christopher J Petzold, Leonard P Guler, Eric D Nelson, and Hilkka I Kenttämaa, "Laser-induced acoustic desorption/chemical ionization in fourier-transform ion cyclotron resonance mass spectrometry," Int. J. Mass Spectrom. 198, 173-188 (2000)

${ }^{25}$ Ryan C Shea, Steven C Habicht, Weldon E Vaughn, and Hilkka I Kenttämaa, "Design and characterization of a highpower laser-induced acoustic desorption probe coupled with a fourier transform ion cyclotron resonance mass spectrometer," Anal. Chem. 79, 2688-2694 (2007)

${ }^{26}$ Ryan C Shea, Christopher J Petzold, J Larry Campbell, Sen Li, David J Aaserud, and Hilkka I Kenttämaa, "Characterization of laser-induced acoustic desorption coupled with a fourier transform ion cyclotron resonance mass spectrometer," Anal. Chem. 78, 6133-6139 (2006).

27 Steven C Habicht, Lucas M Amundson, Penggao Duan, Nelson R Vinueza, and Hilkka I Kenttämaa, "Laser-induced acoustic desorption coupled with a linear quadrupole ion trap mass spectrometer," Anal. Chem. 82, 608-614 (2010)

28 Jinshan Gao, David J Borton, Benjamin C Owen, Zhicheng Jin, Matt Hurt, Lucas M Amundson, Jeremy T Madden, Kuangnan Qian, and Hilkka I Kenttämaa, "Laser-Induced
Acoustic Desorption/Atmospheric Pressure Chemical Ionization Mass Spectrometry," J. Am. Soc. Mass. Spectrom. 22, 531-538 (2011).

${ }^{29}$ C R Calvert, L Belshaw, M J Duffy, O Kelly, R B King, A G Smyth, T J Kelly, J T Costello, D J Timson, W A Bryan, T Kierspel, P Rice, I C E Turcu, C M Cacho, E Springate, I D Williams, and J B Greenwood, "LIADfs scheme for studies of ultrafast laser interactions with gas phase biomolecules," Phys. Chem. Chem. Phys. 14, 6289-6297 (2012)

su Louise Belshaw, Francesca Calegari, Martin J Duffy, Andrea Trabattoni, Luca Poletto, Mauro Nisoli, and Jason B Greenwood, "Observation of ultrafast charge migration in an amino acid," J. Phys. Chem. Lett. 3, 3751-3754 (2012)

31 F Calegari, D Ayuso, A Trabattoni, L Belshaw, S De Camillis, S Anumula, F Frassetto, L Poletto, A Palacios, P Decleva, J B Greenwood, F Martín, and M Nisoli, "Ultrafast electron dynamics in phenylalanine initiated by attosecond pulses." Science 346, 336-339 (2014).

32 Ning Zhang, Kai Zhu, Caiqiao Xiong, Yurong Jiang, HuanCheng Chang, and Zongxiu Nie, "Mass measurement of single intact nanoparticles in a cylindrical ion trap," Anal. Chem. 88, 5958-5962 (2016).

33 Alexander V Bulgakov, Nathan Goodfriend, Oleg Nerushev, Nadezhda M Bulgakova, Sergei V Starinskiy, Yuri G Shukhov, and Eleanor E B Campbell, "Laser-induced transfer of nanoparticles for gas-phase analysis," J. Opt. Soc. Am. B 31, C15-C21 (2014)

${ }^{34}$ N T Goodfriend, S V Starinskiy, O A Nerushev, N M Bulgakova, A V Bulgakov, and E E B Campbell, "Laser pulse duration dependence of blister formation on backradiated Ti thin films for BB-LIFT," Appl. Phys. A $\mathbf{1 2 2}$, 154 (2016)

35 D P DePonte, U Weierstall, K Schmidt, J Warner, D Starodub, J C H Spence, and R B Doak, "Gas dynamic virtual nozzle for generation of microscopic droplet streams," J. Phys. D 41, 195505 (2008)

36 K R Beyerlein, L Adriano, M Heymann, R. Kirian, J Knoska, F Wilde, H. N. Chapman, and S. Bajt, "Ceramic micro-injection molded nozzles for serial femtosecond crystallography sample delivery," Rev. Sci. Instrum. 86, 125104-12 (2015).

ग Nicole Teschmit, Daniel Gusa, Igor Rubinsky, Daniel A Horke, and Jochen Küpper, "Characterizing and optimizing a laser-desorption molecular beam source," J. Chem. Phys. 147, 144204 (2017) arXiv:1706.04083 [physics.chem-ph]

38 P. J. Linstrom and W. G. Mallard, eds., NIST Chemistry WebBook, NIST Standard Reference Database Number 69 (National Institute of Standards and Technology, Gaithersburg MD, 20899, 2017).

39 Leonid V Zhigilei and Barbara J Garrison, "Velocity distributions of molecules ejected in laser ablation," Appl. Phys Lett. 71, 551-553 (1997)

${ }^{40}$ N G Utterback, S P Tang, and J F Friichtenicht, "Atomic and ionic beam source utilizing pulsed laser blow off," Phys Fluids 19, 900-905 (1976)

${ }^{41}$ B Lindner, "On the desorption of electrosprayed organic compounds from supporting metal foils by laser induced pressure waves," Int. J. Mass Spectrom. Ion Proc. 103, 203-218 (1991)

${ }^{42}$ V V Golovlev, S L Allman, W R Garrett, and C H Chen, "Laser-induced acoustic desorption of electrons and ions," Appl. Phys. Lett. 71, 852-854 (1997).

43 Francesca Calegari, David Ayuso, Andrea Trabattoni, 
Louise Belshaw, Simone De Camillis, Fabio Frassetto, Luca Poletto, Alicia Palacios, Piero Decleva, Jason B Greenwood, Fernando Martín, and Mauro Nisoli, "Ultrafast Charge Dynamics in an Amino Acid Induced by Attosecond Pulses," IEEE Journal of Selected Topics in Quantum Electronics 21, 1-12 (2015)

${ }^{44}$ David J Borton, Lucas M Amundson, Matthew R Hurt, Alex Dow, Jeremy T Madden, Garth J Simpson, and Hilkka I Kenttämaa, "Development of a high-throughput laser-induced acoustic desorption probe and raster sampling for laser-induced acoustic desorption/atmospheric pressure chemical ionization," Anal. Chem. 85, 5720-5726 (2013)
45 P A Rigg, R J Scharff, and R S Hixson, "Sound speed measurements in tantalum using the front surface impact technique," J. Phys.: Conf. Ser. 500, 032018 (2014).

46 We evaluated the energy release per molecule based on the known physical constants for anthracene, $\stackrel{48}{~ s i n c e ~ d a t a ~ f o r ~}$ PA was not available. The thermal expansion coefficient of the film is assumed to be $2.8 \times 10^{-4} \mathrm{~K}^{-1}$.

47 Nicholas R Hutzler, Hsin-I Lu, and John M Doyle, "The buffer gas beam: An intense, cold, and slow source for atoms and molecules," Chem. Rev. 112, 4803-4827 (2012)

48 A Bondi, "Thermal properties of molecular crystals. i. heat capacity and thermal expansion," J. Appl. Phys. 37, 46434647 (1966) 